\title{
Acknowledgement of Reviewers
}

The Executive Committee of the International Journal of Medical Students.

The Executive Committee, Editors, and Staff of the International Journal of Medical Students, wish to sincerely thank the following reviewers for their contributions to the Journal and support to medical students worldwide. Your time and expertise are greatly appreciated. Without your contribution, the publication of volume 5 (2017) would not have been possible.

Camelia Cristina Diaconu - Internal Medicine Department, "Carol Davila" University of Medicine and Pharmacy, Bucharest, Romania \& Internal Medicine Clinic, Clinical University Hospital of Bucharest, Bucharest, Romania.

Pishoy Gouda - Division of Internal Medicine, University of Calgary, Calgary, Alberta, Canada.

Eren Tunc - Department of General Surgery, School of Medicine, Coztepe Training and Research Hospital, Istanbul Medeniyet University, Istanbul, Turkey.

Dilia Karina Martínez-Méndez - Immunology Laboratory, Faculty of Experimental Sciences, University of Zulia, Maracaibo, Venezuela.

Cristina Popescu - Infectious Diseases Department, "Carol Davila" University of Medicine and Pharmacy, Bucharest, Romania \& "Prof. Dr. Matei Balş" National Institute for Infectious Diseases, Bucharest, Romania.

Kevin Escandón-Vargas - Faculty of Health, Universidad del Valle school of Medicine, Cali, Colombia.

Siraj Omar Wali - Sleep Medicine and Research Center, King Abdulaziz University Hospital, Jeddah, Saudi Arabia.

Amelia Maria Găman - Pathophysiology Department, University of Medicine and Pharmacy of Craiova, Craiova, Romania \& Hematology Clinic, Filantropia City Hospital, Craiova, Romania.

Ana Maria Vintilă - Medical Semiology Department, "Carol Davila" University of Medicine and Pharmacy, Bucharest, Romania it Internal Medicine Clinic, Colțea Clinical Hospital, Bucharest, Romania.

Ahmed Siddiqi - Department of Orthopedic Surgery, Philadelphia College of Osteopathic Medicine, Philadelphia, Pennsylvania, USA.

Aisha Gharaibeh - Faculty of Medicine, The University of Jordan, Amman, Jordan at Vissum Instituto oftalmológico de Alicante, Universidad Miguel Hernández, Alicante, Spain.

Ana Maria Enciu - Ultrastructural Pathology Laboratory, "Victor Babeș" National Institute of Pathology, Bucharest, Romania; Department of Cellular it Molecular Biology and Histology, "Carol Davila" University of Medicine and Pharmacy, Bucharest, Romania.
Szabolcs Szatmári - Department of Neurology, Clinical County Emergency Hospital, Târgu Mureș, Romania; Department of Neurology, University of Medicine and Pharmacy, Târgu Mureș, Romania.

Omar Y. Mousa - Division of Gastroenterology and Hepatology, Mayo Clinic College of Medicine, Mayo Clinic, Jacksonville, FL, USA.

Alaa Althubaiti - Department of Basic Medical Sciences, College of Medicine, King Saud bin Abdulaziz University for Health Sciences, King Abdullah International Medical Research Centre, Riyadh, Saudi Arabia.

Michael A. Gaudiani - Department of Orthopedic Surgery, Hospital for Special Surgery, New York, New York, USA.

Cristian Răsvan Băicuș - Internal Medicine Department, "Carol Davila" University of Medicine and Pharmacy, Bucharest, Romania at Internal Medicine Clinic, Colentina Clinical Hospital, Bucharest, Romania.

Andrew J. Larner - Walton Centre for Neurology and Neurosurgery, Liverpool, UK.

Paul MacDaragh Ryan - School of Medicine, University College Cork, Cork, Ireland.

Vlad Denis Constantin - Surgery Department, "Carol Davila" University of Medicine and Pharmacy, Bucharest, Romania a Surgery Department, St. Pantelimon Emergency Clinical Hospital, Bucharest, Romania.

Lukas Rasulić - School of Medicine, University of Belgrade, Belgrade, Serbia a Department of Peripheral Nerve Surgery, Functional Neurosurgery and Pain Management Surgery, Clinic for Neurosurgery, Clinical Center of Serbia, Belgrade, Serbia.

Supasit Pannarunothai - Centre for Health Equity Monitoring, Faculty of Medicine, Naresuan University, Thailand.

Bogdan Socea - Surgery Department, "Carol Davila" University of Medicine and Pharmacy, Bucharest, Romania a Surgery Department, St. Pantelimon Emergency Clinical Hospital, Bucharest, Romania.

Álvaro E. Mondragón-Cardona - Internal Medicine Department, Universidad Surcolombiana, Neiva, Colombia. 
Alina Cristina Negut - Infectious Diseases Department, "Carol Davila" University of Medicine and Pharmacy, Bucharest, Romania \& "Prof. Dr. Matei Balş" National Institute for Infectious Diseases, Bucharest, Romania.
Fátima Rodriguez Acosta - Molecular Biology and Biotechnology Department, Clinical Analyses and Microbiology Department, Institute of Investigations in Health Sciences, San Lorenzo, Paraguay.

Ahmed M. Agiba - RAD Department, SICMA Pharmaceutical Industries, Egypt \& Ain Shams University, Cairo, Egypt. 